\title{
High Performance Geocomputation - Preface
}

\author{
Yong Xue ${ }^{1,2, *}$, Dingsheng $\mathrm{Liu}^{3}$, Jianwen $\mathrm{Ai}^{1,4}$, and Wei Wan ${ }^{1,4}$
}

\begin{abstract}
${ }^{1}$ State Key Laboratory of Remote Sensing Science, Jointly Sponsored by the Institute of Remote Sensing Applications of Chinese Academy of Sciences and Beijing Normal University, Institute of Remote Sensing Applications, CAS, P.O. Box 9718, Beijing 100101, China

${ }^{2}$ Department of Computing, London Metropolitan University, 166-220 Holloway Road, London N7 8DB, UK

${ }^{3}$ Center for Earth Observation and Digital Earth, Chinese Academy of Sciences, Beijing 100080, P.R. China

${ }^{4}$ Graduate University of Chinese Academy of Science, Yuquan Road, Beijing 100049, China y.xuellondonmet.ac.uk
\end{abstract}

\begin{abstract}
This paper presents the introduction to Geocomputation workshop in ICCS2008. The Workshop on Geocomputation continues with the ICCS conferences held in Amsterdam (2002), St. Petersburg (2003), Krakow (2004), Atlanta (2005), Reading (2006), and Beijing (2007).
\end{abstract}

\section{Preface}

High Performance Geo-Computation (HPGC) is the application of high performance computational science to Earth sciences. It applies high-performance computational resources to various types of Earth science data, information, and models for solving Earth science problems. It develops discipline-specific theories, algorithms, architectures, systems, supporting tools, and infrastructure within the overall context of computational science. HPGC is concerned with new computational techniques, algorithms, and paradigms that are dependent upon and can take advantage of high performance computing, distributed computing, and high throughput computing. The areas of application for HPGC include, but are not limited to, spatial data analysis, dynamic modelling, simulation, space-time dynamics and visualization, virtual reality, and applications employing non-conventional data clustering and analysis techniques. HPGC is an integrated computing environment. It is driven by the advances in computing technologies, such as cluster computing, the pervasive computing and/or ubiquitous computing and Grid computing as part of a common framework that offers the best immersion of users and applications in the global environment.

However, the key technologies for Geo-grid differ from those for general purpose Grid computing in many aspects, which often refer to methods and solutions being used to implement geo scientific application on top of grid infrastructure. These technologies include three levels:

\footnotetext{
${ }^{*}$ Corresponding author.
} 
1. The Grid adaptation to geoscience, Grid enabled geo resources, and Grid enabled geographic application. The Grid adaptation to geoscience, namely incorporating general-purpose Grid middleware and third party components into Earth science missions, aims to change the Grid to better meet the needs from geo community. For example, traditional Grid service for data management are required to be modified, as for adapting to complexity and massiveness in spatial data processing, by modifying the data transfer protocol and improving data replica mechanism.

2. Grid enabled geo resources means builders and administrators change diverse resources of geo-spatial data, models and algorithms, with special emphasis on encapsulating, deploying and registering them in the grid environment. Thus users can have access to these resources. Grid enabled geo resources is one of the most important parts with respect to Geo-Grid.

3. Grid enabled geographic application actually is a sets of invocations of operations with dependency on each other, and the contexts in which certain operations are. Grid application in geo science filed include as follow: resources describing mechanism, platform building-up tool, visualization tool, and develop tool. These toolboxes provide essential foundation for building large-scale system for Geo-science application.

This workshop consists of six papers. All papers have gone through the normal refereeing process of ICCS conference. These papers discuss recent results on several aspects of geocomputation, including theories and applications. The first paper by Jankowski et al. deals with the numerical simulation of threshold-crossing problem allowing us to assess the probability that a random field of contamination does not exceed a fixed level in a certain two-dimensional (2-D) spatial domain. The numerical evaluation of the Legendre functions are especially challenging for very high degrees and orders which are required for advanced geocomputations. The computational aspects of SHTs and their inverses using both quadrature and leastsquares estimation methods are discussed with special emphasis on numerical preconditioning that guarantees reliable results for degrees and orders up to 3800 in REAL*8 or double precision arithmetic in the paper by Blais et al.. In the paper from Tawfik et al., a prototype, which uses context information and a range of design and user-related criteria to analyse the accessibility of road layouts and plan routes, is developed; a case study is used to validate the prototype. In the paper by Cope et al., authors examined emerging urgent Earth and geoscience workflows, the data services used by these workflows, and their proposed urgent data management framework for managing urgent data services. The design, analysis and implementation of InterCondor system was presented in the paper by Xue et al.. The InterCondor system is an implementation of the concept of InterGrid. Finally, Liu et al. in their paper proposed the new architecture of SIG, upon which the constituted GIS nodes can provide GISer-vices based on existing SIG platform.

Last but not least, we are grateful to the authors and referees for their hard work. 